\title{
SEM Image Observation Using an Electron Energy and Electron Take-off Angle Filtered Detector
}

\author{
Takeshi Otsuka $^{1}$, Motohiro Nakamura ${ }^{1}$, Ken-ichi Yamashita ${ }^{1}$, Masaya Hara ${ }^{1}$, Felix Timischl ${ }^{2}$, Kazuhiro \\ Honda $^{1}$, Masato Kudo ${ }^{2}$ and Shin-ichi Kitamura ${ }^{1}$ \\ 1. JEOL Ltd., 1-2 Musashino, 3-Chome, Akishima, Tokyo, 196-8558, Japan \\ 2. JEOL Technics Ltd., 6-38 Musashino 2-chome, Akishima, Tokyo, 196-0021, Japan
}

Scanning electron microscopes (SEMs) are usually equipped with two types of detectors: secondary and backscattered electron detectors. The former produces secondary electron images (SEI) rich in topographic information[1, 2], whereas the latter produces backscattered electron images (BEI) rich in composition information[3]. Recently, however, a few other detectors have been installed in addition to these two types of conventional detectors. For example, four different detectors can be installed in a field emission SEM JSM-7800F: an upper electron detector (UED), an upper secondary electron detector (USD), a backscattered electron detector (BED), and a lower electron detector (LED). They allow to separate electron energy and take-off angle. Therefore, they help to observe specimen shape and structure on nano-scale[4]. On the other hand, they detect electrons having passed through the electromagnetic field of the objective lens, which changes actual energy and take-off angle ranges. Consequently, it is difficult to distinguish the image contrasts in accordance with physical properties. In this study, an electron detector was designed and experimentally manufactured to detect electrons emitted in a definite, variable range of energy and take-off angle.

In practical detectors it is difficult to see directly the effect of energy and take-off angle of the emitted electrons on the image contrast.

Figure 1 shows a schematic diagram of the newly designed electron detector, the E- $\theta$ detector, which can detect electrons emitted from a sample with a selected range of energy and take-off angle. The E- $\theta$ detector consists of a slit plate, inner and outer electrodes in a cage, and an electron detector. The slit plate is placed at a lower part of the E- $\theta$ detector. It serves as a selector of take-off angle. The range of take-off angle is selected mechanically by sliding the slit plate as shown in Fig. 1 and is measured from the horizontal direction parallel to the sample surface. The selection of electron energy is achieved by applying voltage to the inner and outer electrodes in the cage in accordance with electron's take-off angles. In the case of the low angle range, positive and negative voltages are applied to the inner and outer electrode, respectively, as shown in Fig. 1(A), so that electrons are deflected towards the inside with the increasing amount of deflection with decreasing energy. In the case of the high angle range, the sign of the applied voltage is reversed as shown in Fig. 1(B), so that electrons are deflected towards outside. To enhance the electron detection, a metal mesh is placed in front of the electron detectors and a voltage of $2 \mathrm{keV}$ is applied between the detectors and the metal mesh. When a series of concentric ring electron detectors with different diameters are placed at the upper part of the cage, each ring detector can collect electrons with a specific range of energy determined by the voltage applied to the two electrodes. As a preliminary study, we acquired images using commercial Si-photodiode (SiPD) as shown Fig. 2. In this case, the SiPD is a square shape instead of concentric rings. Therefore, we aligned SiPD detectors in the radial direction. 
We are now manufacturing a new SiPD electron detector for the E- $\theta$ detector. It divides into seven sections in the radial direction. In the presentation, we will be able to show series of images taken with different ranges of energy and take-off angle.

[1] M. Kotera et al, Scanning Microscopy Supplement 4 (1990) p. 111.

[2] Y. Lin and David C. Joy, Surface and Interface Analysis 37 (2005) p. 895.

[3] M. D. Ball, D. G. McCartney, Journal of Microscopy 124 (1981) p. 57.

[4] S. Asahina et al, Microscopy and Analysis, Nanotechnology supplement November (2012)

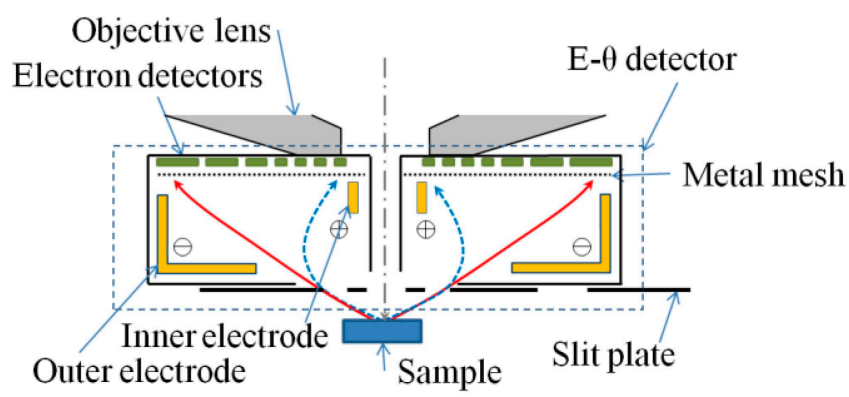

A) For the low angle range

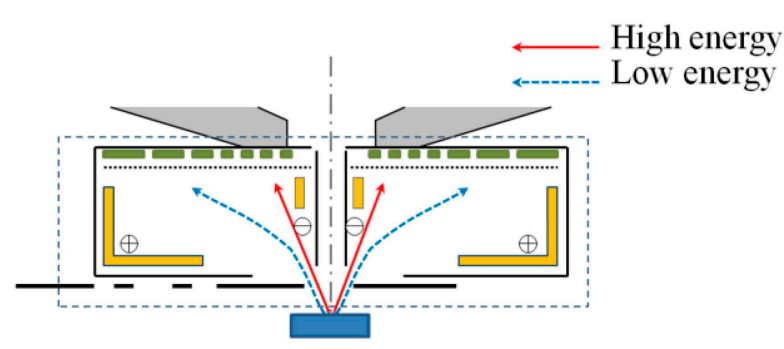

B) For the high angle range

Figure 1. Schematic diagram of the E- $\theta$ detector.

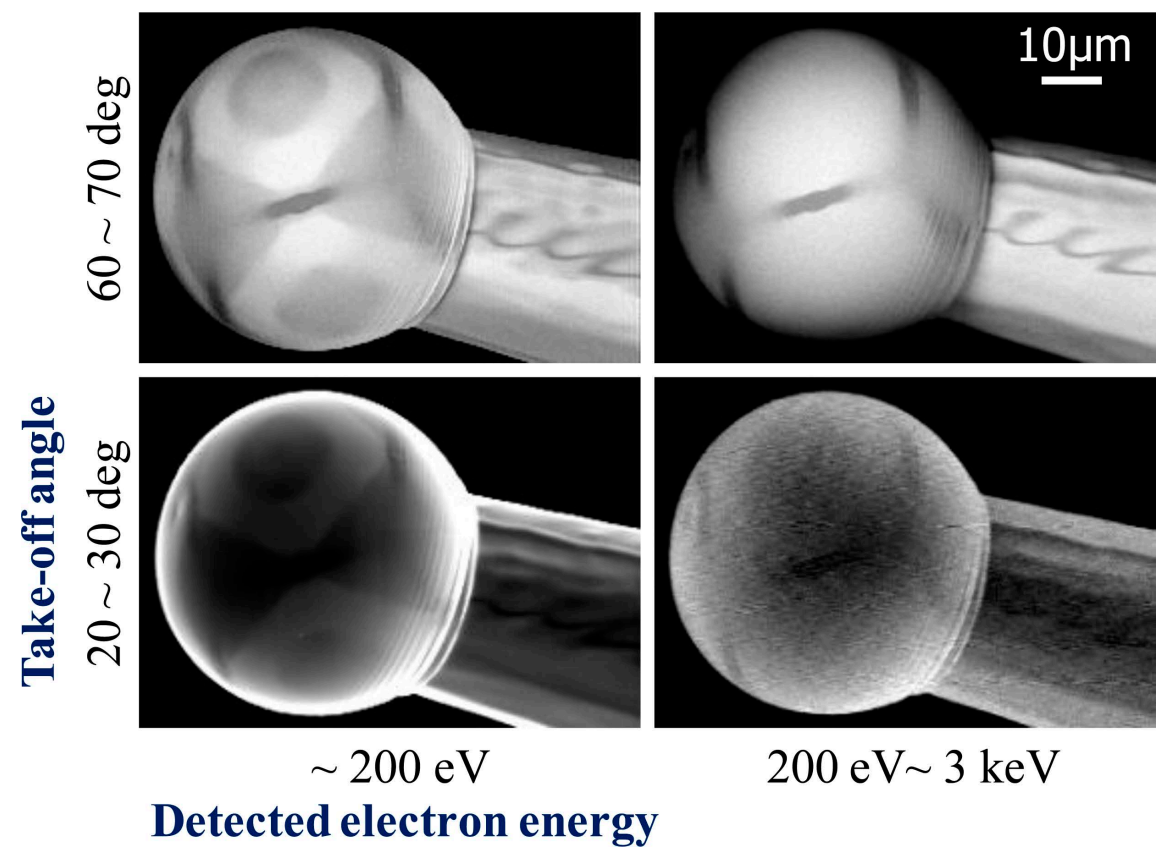

Figure 2. SEM images acquired with the E- $\theta$ detector. The sample is a spherical single crystal of tungsten. 